\title{
Perspektiven der ökonomischen Bewertung von Ökosystemleistungen
}

\author{
Die Nutzung der Ergebnisse ökonomischer \\ Bewertungen von Ökosystemleistungen spielt \\ bei Entscheidungen im Umwelt- und Naturschutz \\ in Deutschland keine Rolle. Bessere Kenntnisse \\ in Wissenschaft und Politik sowie eine Methoden- \\ konvention könnten diese Situation ändern. \\ Von Jürgen Meyerhoff und Ulrich Petschow
}

\footnotetext{
Ö
} konomische Ansätze haben in den vergangenen Jahren in der Umweltpolitik an Relevanz gewonnen. Im Kontext der Europäischen Union (EU) gilt dies zum Beispiel für verschiedene Politikbereiche (unter anderem Biodiversitätspolitik) oder konkret für Direktiven (EG-Wasserrahmenrichtlinie oder die EG-Meeresschutzstrategie-Richtlinie). Auch im Rahmen internationaler Abkommen spielen ökonomische Ansätze eine zunehmende Rolle, so im Kontext der Biodiversitätskonvention (CBD). Die ökonomische Bewertung spielt sowohl für die Klimapolitik als auch für die Naturschutz- und Biodiversitätspolitik eine zunehmende Rolle. Der mediale Erfolg des Stern-Reports zu den Kosten des unterlassenen Klimaschutzes hat Anstoß gegeben für die internationalen TEEB-Studien, die aufbauend auf dem Millennium Ecosystem Assessment (MEA) durch die systematische Erfassung von Ökosystemleistungen (ÖSL) auch die ökonomische Relevanz von Natur und Landschaft und den damit verbundenen ÖSL aufzeigt. Durch diese Entwicklungen werden die ökonomischen Werte zumindest medial zu einem Argument für den Klima- und Biodiversitätsschutz.

Inhaltlich fokussieren die TEEB-Studien, sowohl die internationalen als auch die deutschen, auf eine Zusammenführung und Darstellung existierender ökonomischer Bewertungsstudien, um sie unter anderem auch einer breiteren Öffentlichkeit zugänglich zu machen. In diesem Zusammenhang sind zwei Herausforderungen sehr deutlich geworden: Erstens ist die empirische Basis, und dies gilt insbesondere für Deutschland, sehr dünn sowohl hinsichtlich der angewendeten Methoden als auch hinsichtlich der Bewertungsobjekte (ÖSL). Zweitens besteht Handlungsbedarf hinsichtlich der Nutzung der Ergebnisse aus Studien zum ökonomischen Wert von Natur und Landschaft. Ein Blick in die Methodik der Nutzen-Kosten-Analysen (NKA) zum Beispiel im Rahmen der Bundesverkehrswegeplanung zeigt, dass hier in den letzten 30 Jahren nicht viel Fortschritt zu verzeichnen ist (Meyerhoff/Petschow 1995).

\section{Methodische Herausforderungen}

Obwohl die Agenda zum Wert von ÖSL ihren Ausgangspunkt mit in der Ökonomik hat, sind vor allem in Deutschland die Diskussionen durch eine starke Skepsis, wenn nicht gar Ablehnung, der ökonomischen Bewertung von Umweltveränderungen und der dafür entwickelten Methoden geprägt (zu den Methoden siehe Hanley/Barbier, 2009). Zur Begründung werden in der Regel zwei Argumente vorgebracht. Zum einen wird darauf verwiesen, dass die Ergebnisse, insbesondere wenn sie auf umfragebasierte Studien zur Zahlungsbereitschaft zurückgehen, wenig belastbar und vertrauenswürdig seien. Zum anderen wird hervorgehoben, dass der Schutz von Natur und Landschaft (und Biodiversität) bereits weitgehend rechtlich und planerisch geregelt ist und damit die Relevanz der ökonomischen Bewertung fraglich ist. Beide Argumente haben etwas für sich, fußen allerdings einerseits häufig auf vor-analytischen Vorstellungen und geringem Wissen über Anliegen und Methoden der ökonomischen Bewertung. Andererseits wurden die Bewertungsmethoden in den vergangenen Jahren deutlich weiterentwickelt und viele der Punkte, die heute gerne noch von Kritikern bemüht werden, sind in der Form oft nicht mehr zutreffend.

Bedenkt man weiterhin, dass der Bedarf an ökonomischen Bewertungen von Umweltveränderungen und ÖSL angesichts der zunehmenden Relevanz nicht allein im Kontext von EU-Regulierungen steigen wird, gleichzeitig aber kein konsistentes Forschungs- und Entwicklungskonzept besteht, um diesen Herausforderungen zu begegnen, dann stellt sich die Frage nach den Perspektiven der ökonomischen Bewertung in Deutschland. Vor diesem Hintergrund sollen im Folgenden Konturen eines Programms zur Verbesserung sowohl der Methodenkompetenz als auch der Kenntnisse über den Wert von Ökosystemleistungen aufgezeigt werden. Die zentrale Frage dabei ist, in welchem Umfang Methodenkompetenz im Bereich der ökonomischen Bewertung von Ökosystemleistungen in Deutschland aufgebaut werden soll und Beiträge zur Methodenentwicklung geleistet werden sollen. Vor dem Hintergrund auch der oben angesprochenen politischen Dynamiken, insbesondere auf EU-Ebene, erscheint es geboten, die Forschung in diesem Bereich zu stärken. Sie gehört international nach wie vor zu einem der lebhaftesten Bereiche der Umweltökonomik und der ökologischen Ökonomik, hat aber auch in Nachbardisziplinen wie der Transportökonomik oder Gesundheitsökonomik eine hohe Relevanz. Dies gilt insbesondere für den Bereich der umfragebasierten Bewertungsmethoden. Welche Bedeu- 
tung die Methodenentwicklung in der Wissenschaft hat, belegt die hohe Anzahl an Veröffentlichungen in den entsprechenden Journals. Ein Blick in diese Journals zeigt aber auch, dass der Beitrag aus Deutschland hierzu sehr gering ist. Um diese Situation zu ändern, aber auch um das Wissen über den ökonomischen Wert von ÖSL zu vergrößern, könnten folgende Schritte hilfreich sein:

\section{Entwicklung von Methoden}

Es sollten Forschungsinitiativen, sei es vom Bundesministerium für Bildung und Forschung oder vom Bundesministerium für Umwelt, Naturschutz, Bau und Reaktorsicherheit, aufgelegt werden, die explizit die Forschung zu den Bewertungsmethoden zum Gegenstand haben. Bisher ist die ökonomische Bewertung von ÖSL in der Regel ein Teilvorhaben in interdisziplinären Forschungsprojekten und soll dort Zahlen zum ökonomischen Wert ausgewählter Dienstleistungen, die vielfach aus eher projektspezifischen Fragestellungen abgeleitet werden, liefern. Dieses Vorgehen hat sicher in vielen Fällen eine Berechtigung, trägt aber nicht dazu bei, dass methodische Probleme systematisch bearbeitet werden. Hierfür wäre es Voraussetzung, dass zum Beispiel explizit mit Splitsamplen gearbeitet werden kann, um den Einfluss verschiedener Treatments testen zu können. Dies widerspricht aber zum Teil der Anforderung in angewandten Forschungsvorhaben, die „eine“ Zahl für den Wert einer bestimmten Ökosystemleistung zu liefern. Weiterhin ist ein stark interdisziplinäres Umfeld nicht immer die beste Voraussetzung für die Methodenentwicklung, da diese durchaus disziplinären Austausch und auch Auseinandersetzungen erfordert.

Bedarf bei der Methodenentwicklung besteht sowohl bei den indirekten als auch den direkten Methoden. Interessanterweise werden gerade die indirekten Methoden als diejenigen angesehen, die validere Ergebnisse ermöglichen. Dies wird damit begründet, dass die Methoden auf beobachtbarem Verhalten beruhen und daher eine solidere Basis haben als die direkten Methoden, die auf Umfragen beruhen. Häufig wird dabei verkannt, dass die Verwendung der indirekten Methoden eine Vielzahl von Annahmen voraussetzt, um die „beobachteten“ Daten für die Ableitung ökonomischer Werte nutzen zu können. Auch beruhen viele Studien, die indirekte Methoden einsetzen, auf Interviews, in denen zum Beispiel zurückliegende Besuche einer Landschaft und deren Einschätzung erfragt werden. Es handelt sich dabei also keinesfalls um beobachtetes Verhalten, sondern eher um „erinnertes Verhalten“.

Die möglichen Themen für die Methodenforschung sind vielfältig und können hier nur unvollständig angedeutet werden. Im Bereich der direkten Bewertungsverfahren gehört hierzu sicher der Embedding Effekt (Hansjürgens 2014), andere zur Zeit stärker diskutierte Themen sind die Consequentiality, d. h. wie stark ein Zusammenhang zwischen Umfrageergebnissen und Politikentscheidungen gesehen wird, die Anwendung von Heuristiken bei der Beantwortung von Choice-
Experimenten oder der räumliche Kontext im Sinne der Verfügbarkeit von Substituten oder die Distanz zum Bewertungsobjekt. Für einen Überblick über Forschungsthemen im Zusammenhang mit der Anwendung von Choice-Experimenten siehe Adamowicz et al. (2014). Diese Methode wird zunehmend auch für die Bewertung der Erholungsfunktion von Landschaften eingesetzt. Im Bereich der indirekten Methoden erlebt die hedonische Bewertung eine gewisse Renaissance, gerade im Bereich städtischen Grüns. Themen für die Forschung sind hier der Einsatz sogenannter Sorting-Modelle sowie die Kombination der hedonischen Analyse mit Discrete Choice-Modellen (Phaneuf et al. 2013).

\section{Verbreiterung der empirischen Basis}

Weitere empirische Studien werden benötigt. Die Zahl der in Deutschland durchgeführten Studien ist bislang sehr überschaubar, die vorliegenden Studien beziehen sich zudem in der Regel auf einzelne Aspekte des Naturschutzes wie z. B. den Schutz einer einzelnen Art oder einzelnen Landschaft. Insbesondere im Hinblick auf die Nutzung des Benefit-Transfers ist es jedoch erforderlich, eine größere Zahl an empirischen Studien zur Verfügung zu haben, um zum Beispiel auch MetaAnalysen durchführen zu können. Ansonsten gilt: Wo nichts ist, kann auch nichts übertragen werden. Zu weiteren Aspekten der Nutzung des Benefit-Transfers für die ökonomische Bewertung von Ökosystemleistungen siehe Richardson et al. (2014).

Die Berichte im Rahmen des TEEB Deutschland zeigen sehr deutlich, dass häufig immer wieder auf wenige Studien zurückgegriffen wird, wenn es um die Darstellung von Fallbeispielen zur ökonomischen Bewertung geht. Ein Beispiel hierfür sind die Arbeiten, die im Zusammenhang mit der Elbeforschung erstellt wurden (siehe Grossmann et al. 2010). Sie sind zwar gut geeignet als Beispiele für die Anwendung der ökonomischen Bewertung, sind aber inzwischen doch in die Jahre gekommen und erstrecken sich nur auf Teile des gesamten ökonomischen Wertes. So treffen diese Studien keine Aussagen über den Erholungswert der Auen an der Elbe, dieser wurde nicht untersucht. Gerade im Hinblick auf den Erholungswert der Landschaft in Deutschland auch jenseits von Feuchtgebieten sind empirische Untersuchungen Mangelware. Methodisch gute Arbeiten sind kaum vorhanden und die bekannten Ausnahmen (Grossmann 2012, Lienhoop/Ansmann 2011) bestätigen höchstens die Regel, dass in einem Land, in dem die Landschaft allein schon aufgrund der hohen Bevölkerungsdichte eine besondere Bedeutung für die Erholung hat, nicht viel über ihren Wert bekannt ist. Interessant an der Rezeption der Studie von Grossmann et al. (2010) ist zudem, dass gerade sie zeigt, dass die für den Naturschutz beste Form des Hochwasserschutzes nur dann eindeutig wirtschaftlich ist, wenn die Ergebnisse einer Zahlungsbereitschaftsanalyse für den Natur- und Landschaftsschutz an der Elbe berücksichtigt werden. Dieses „Detail“ wird aber bei der Präsentation der Ergebnisse gerne übersehen. Für die Nutzung in der Umwelt- und Naturschutzpolitik könnten 
insbesondere Studien hilfreich sein, die umfangreichere Programme zum Beispiel zum Naturschutz bewerten. Ein frühes Beispiel für diesen Ansatz ist die Studie von Hampicke et al. (1991), die ein Naturschutzprogramm für Deutschland bewertet haben. Diesem Vorbild sind Meyerhoff et al. (2013; siehe auch Wüstemann et al. 2014) gefolgt.

Die vorhandenen Daten (bzw. auch durch ein entsprechendes Forschungsprogramm zu generierende Daten) sollten so zusammengestellt und aufbereitet werden, dass sie für Interessierte und Entscheidungsträger leicht zugänglich sind und zudem für den Einsatz im Rahmen von Benefit-TransferStudien genutzt werden können. Die Anlage einer Datenbank wäre hier ein guter Anfang. Erstaunlicherweise war die Anlage einer solchen Datenbank im Kontext der deutschen TEEB-Studien nicht vorgesehen, obwohl diese doch gerade auf vorhandenen Studien aufbauen sollen.

\section{Entwicklung einer Methodenkonvention}

Für die Nutzung der Ergebnisse aus ökonomischen Bewertungsstudien in konkreten Entscheidungsverfahren, zum Beispiel Kosten-Nutzen-Analysen für Investitionen des Bundes in den Ausbau von Flüssen, wäre es hilfreich, wenn nach Durchführung eines Forschungsprogramms „Weiterentwicklung der Methoden und systematische Erfassung der Ökosystemleistungen" eine Konvention entwickelt würde, die Anforderungen an Bewertungsstudien definiert. Es geht dabei um die Entwicklung von Richtlinien, mit deren Hilfe festgelegt werden sollte, welche Veränderungen in Natur und Landschaft mit welchen Methoden bewertet werden sollten oder welche Erwartungen an die statistische Sicherheit der Schätzungen seitens der Entscheidungsträger gestellt werden. Das Umweltbundesamt (2013) hat im letzten Jahr eine aktualisierte Methodenkonvention "Ökonomische Bewertung von Umweltschäden“ herausgegeben. Diese Methodenkonvention ist allerdings auf die Bewertung der Folgeschäden von Kohlendioxid und Luftschadstoffen fokussiert. Daher sollte diese vorliegende Konvention um einen Teil zur ökonomischen Bewertung von Ökosystemleistungen im Bereich Natur und Landschaft erweitert werden.

\section{Fazit}

Der Artikel versucht, für die ökonomische Bewertung von Ökosystemleistungen oder, traditioneller formuliert, für Veränderungen in Natur und Landschaft, einige Schritte zu benennen, mit denen der heutige geringe Kenntnisstand in Deutschland sowohl hinsichtlich der Methoden als auch der Kenntnisse über die ökonomischen Werte verbessert werden könnte. Ein entsprechendes Programm erscheint in Deutschland überfällig. Mit Blick auf die Praxisrelevanz ist allerdings offen, ob Entscheidungsträger tatsächlich von dieser Art von Informationen Gebrauch machen und die Ergebnisse ökonomischer Bewertungen zum Beispiel auch in Kosten-Nutzen-Analysen berücksichtigt werden (vgl. auch Dehnhardt in diesem Heft). Man bedenke, wie viele Entscheidungen gerade im Bereich staatlicher Infrastrukturinvestitionen pro Ausbau gefallen sind, obwohl ökonomische Kosten-Nutzen-Vergleiche hierfür kein Argument geliefert haben. Wie heißt es doch heute in Teilen von Bayern: Hoi, a Schiff!

\section{Literatur}

Adamowicz, W. L./Glenk, K./Meyerhoff, J. (2014): Choice Modelling Research in Environmental and Resource Economics. In: Hess, S./Daly, A.: Handbook of Choice Modelling, Edward Elgar (in press).

Grossmann, M. (2011). Impacts of boating trip limitations on the recreational value of the Spreewald wetland: a pooled revealed/contingent behaviour application of the travel cost method. In: Journal of Environmental Planning and Management 54, 2/2011. S. 211-226.

Grossmann, M./Hartje, V./Meyerhoff, J. (2010): Ökonomische Bewertung naturverträglicher Hochwasservorsorge an der Elbe. Naturschutz und Biologische Vielfalt 89, Bundesamt für Naturschutz: Bonn.

Hampicke, U./Horlitz, H./Kiemstedt, K./Tampe, K./Timp, D./Walters, M. (1991): Kosten und Wertschätzung des Arten- und Biotopschutzes. Berlin (UBA Berichte 3/91).

Hanley, N./Barbier, E. B. (2009): Pricing Nature. Cost-Benefit Analysis and Environmental Policy, Cheltenham.

Hansjürgens, B. (2014): Ökosystemleistungen und ihre ökonomische Bewertung im Visier von Politik und Forschung in Deutschland. In: Natur und Landschaft 02/2014. S. 56-61.

Lienhoop, N./Ansman, T. (2011): Valuing water level changes in reservoirs using two stated preference approaches: An exploration of validity. In: Ecological Economics 70. S. 1.250-1.258.

Meyerhoff, J./Angeli, D./Hartje, V. (2012): Valuing the benefits of implementing a national strategy on biological diversity - the case of Germany. In: Environmental Science \& Policy 23. S. 109-119.

Meyerhoff, J./Petschow U. (1995): Natur und Umwelt in der Kosten-NutzenAnalyse der Bundesverkehrswegeplanung. In: Zeitschrift für angewandte Umweltforschung 8, 4/1995. S. 544-556.

Phaneuf, D. L./Taylor, L./Braden, J. (2013): Combining RP and SP data to estimate preferences for residential amenities: a GMM approach. In: Land Economics 89. S. 30-52.

Richardson, L./Loomis, J./Kroeger, T./Casey, F. (2014): The role of benefit transfer in ecosystem service valuation. In: Ecological Economics (in press).

Umweltbundesamt (Hrsg.) (2013): Ökonomische Bewertung von Umweltschäden. Methodenkonvention 2.0 zur Schätzung von Umweltkosten. Dessau-Roßlau.

Wüstemann, H./Meyerhoff, J./Rühs, M./Schäfer, A./Hartje, V. (2014): Financial costs and benefits of a program of measures to implement a National Strategy on Biological Diversity in Germany. In: Land Use Policy 36. S. 307-318.

\section{AUTOREN + KONTAKT}

Jürgen Meyerhoff ist wissenschaftlicher Mitarbeiter am Fachgebiet Landschaftsökonomie der Technischen Universität Berlin.

TU Berlin, Fachgebiet Landschaftsökonomie, Straße des 17. Juni 145, 10623 Berlin.

E-Mail: juergen.meyerhoff@tu-berlin.de

Ulrich Petschow ist Leiter des Forschungsfeldes Umweltökonomie und Umweltpolitik am Institut für ökologische Wirtschaftsforschung (IÖW). IÖW, Potsdamer Str. 105, 10785 Berlin. Tel.: +49 30 884594-0, E-Mail: ulrich.petschow@ioew.de, Website: www.ioew.de 